\title{
Ultrasmall gold nanorod-polydopamine hybrids for enhanced photoacoustic imaging and photothermal therapy in second near-infrared window
} \author{
Jin $^{2}$, Matthew Creyer ${ }^{2}$, and Jesse V. Jokerst $1,2,4, \square$ \\ 1. Materials Science and Engineering Program, University of California San Diego, La Jolla, California, 92093, United States. \\ 2. Department of Nanoengineering, University of California San Diego, La Jolla, California, 92093, United States. \\ 3. Department of Bioengineering, University of California San Diego, La Jolla, California, 92093, United States. \\ 4. Department of Radiology, University of California San Diego, La Jolla, California, 92093, United States. \\ $\bowtie$ Corresponding author: jjokerst@ucsd.edu
}

Wonjun Yim ${ }^{1}$, Raina M. Borum², Jiajing Zhou², Yash Mantri ${ }^{3}$, Zhuohong Wu², Jingcheng Zhou ${ }^{2}$, Zhicheng

(c) The author(s). This is an open access article distributed under the terms of the Creative Commons Attribution License (https://creativecommons.org/licenses/by/4.0/). See http://ivyspring.com/terms for full terms and conditions.

Received: 2021.06.07; Accepted: 2021.06.19; Published: 2022.01.01

\begin{abstract}
Gold nanorods (GNRs) have attracted great interest for photo-mediated biomedicines due to their tunable and high optical absorption, high photothermal conversion efficiency and facile surface modifiability. GNRs that have efficient absorption in second near-infrared (NIR-II) window hold further promise in bio-applications due to low background signal from tissue and deep tissue penetration. However, bare GNRs readily undergo shape deformation (termed as 'melting effect') during the laser illumination losing their unique localized surface plasmon resonance (LSPR) properties, which subsequently leads to PA signal attenuation and decreased photothermal efficiency. Polydopamine (PDA) is a robust synthetic melanin that has broad absorption and high photothermal conversion. Herein, we coated GNRs with PDA to prepare photothermally robust GNR@PDA hybrids for enhanced photo-mediated theranostic agents. Ultrasmall GNRs (SGNRs) and conventional large GNRs (LGNRs) that possess similar LSPR characteristics as well as GNR@PDA hybrids were compared side-by-side in terms of the size-dependent photoacoustic (PA) imaging, photothermal therapy (PTT), and structural stability. In vitro experiments further demonstrated that SGNR@PDA showed 95\% ablation of SKOV3 ovarian cancer cells, which is significantly higher than that of LGNRs (66\%) and SGNRs (74\%). Collectively, our PDA coating strategy represents a rational design for enhanced PA imaging and efficient PTT via a nanoparticle, i.e., nanotheranostics.
\end{abstract}

Key words: miniature gold nanorod, core-shell structure, synthetic melanin, second near-infrared

\section{Introduction}

Nanotechnology has significantly advanced the development of bioimaging and light-activated phototherapy such as photoacoustic (PA) imaging and photothermal therapy (PTT) [1, 2]. In PA imaging, a pulsed laser illuminates biological tissues or a contrast agent, and the absorbed light energy is converted into heat energy thereby leading to transient expansion of the absorber and generating acoustic waves detected via ultrasonic transducers [3]. PA imaging is noninvasive and offers high spatial resolution, deep penetration, and fast imaging speed.
It has attracted immense attention of researchers for biomedical imaging [4-6]. Both endogenous (e.g., hemoglobin or melanin) and exogenous (e.g., plasmonic nanoparticles) contrast agents can be used for PA imaging to monitor specific biological processes or to improve imaging depth and quality in tissue [7].

PTT is a spatiotemporally controllable cancer treatment that has shown great promise in the cancer therapy [8]. Like PA imaging, PTT is based on the conversion of light into heat, but it uses heat for 
thermal ablation against cancer cells. Compared to other conventional methods including radio- or chemo-therapy, PTT is attractive because of high inherent specificity, low cost, and limited invasiveness [9]. For example, PTT causes limited damage to the surrounding healthy tissues because thermal effect occurs only in the presence of PTT agents [10]. Currently, the research topics on photothermal theranostics has expanded rapidly, which greatly benefits from the advances made in novel nanomaterials design, including inorganic, organic, and hybrid nanoparticles [11-14].

Particularly, gold nanorods (GNRs) are widely investigated for both PA imaging and PTT due to their high absorption efficiency, photothermal conversion efficiency, and low toxicity. Moreover, the localized surface plasmonic resonance (LSPR) of GNRs is easily tunable by controlling their aspect ratio. GNRs with high aspect ratio show optical absorption in second near-infrared (NIR-II) window $(1000-1350 \mathrm{~nm})$ which offers deep penetration of light, low background noises from the tissue, and maximum permission exposure (MPE) compared to NIR-I window $(650$ - $950 \mathrm{~nm})$ [15-17]. However, pristine GNRs have a significant limitation: laserinduced shape deformation from thermodynamic instability. Under laser irradiation, the thermodynamic tendency to lower the surface energy makes the rod-like GNRs form nanospheres with greater stability (reduced surface area to volume ratio) [18]. Specifically, it is found that a high-pulsed laser induces both point and line defects that grow into planar defects in the interior of GNRs [19]. These defects consequentially convert GNR $\{110\}$ facets into the energetically stable $\{100\}$ and $\{111\}$ facets to minimize surface energy [18]. As a result, GNRs lose their NIR absorption peak due to the structural deformation thus attenuating PA signal and photothermal efficiency. Therefore, improving thermal stability of GNR could improve the potential use of GNR in photo-mediated applications.

Size reduction is one strategy that can improve photothermal stability. Recently, Emelianov and Gambhir et al. reported a seedless method to synthesize ultrasmall GNRs (SGNRs) [20]. These SGNRs are $50 \%$ smaller than conventional large GNRs (LGNRs), but they maintained a comparable aspect ratio and demonstrated 3-fold PA enhancement due to their higher surface-to-volume ratio. More interestingly, these SGNRs were more photothermally stable than LGNRs after laser irradiation [20]. Nevertheless, further GNR miniaturization for thermal stability optimization is difficult; proper synthesis relies on a wide set of factors that can influence particle growth like choice in concentration, surfactant, reducing agent, and $\mathrm{pH}$. Alternatively, core - shell nanostructures can provide further improvement in photothermal stability and performance of SGNRs. Many groups have investigated a variety of materials such silica, metal oxide, and polymers as coatings for GNRs [21-24]. However, the inherent properties of many of these coatings limit their use in biomedical applications. For example, polysarconsine-coated GNRs show high colloidal stability, but they have poor surface modification [25]. $\mathrm{CuO}$ or $\mathrm{MnO}_{2}$-coated GNRs have facile surface tunability but suffer from cytotoxicity $[26,27]$. In contrast to these methods, we used polydopamine (PDA) to protect pristine GNR probe from the shape deformation. PDA coatings are robust, biocompatible, and surface functionalization [28, 29]. We thus hypothesized that PDA is a pragmatic choice as a GNR shape-preserving coating for theranostic applications.

PDA is a synthetic melanin that is a highly crosslinked structure with broadband absorption. Its abundant catechol moieties accommodate a wide class of noncovalent interactions (e.g., hydrophobic, electrostatic, hydrogen bonding, and $\Pi-\Pi$ stackings) with diverse materials (e.g., inorganic, organic, and protein) [30, 31]. We previously validated PDA coatings improve PA performance and thermal stability of pristine LGNR [32]. However, a critical size limit of nanoparticles is $\sim 100 \mathrm{~nm}$ for in vivo applications [33]. For example, FDA-approved anticancer nanomedicines (e.g., doxorubicin and paclitaxel) which are in size range of $100 \sim 200 \mathrm{~nm}$ showed limited accumulation and penetration in tumors [34, 35], while nanomedicines with the size below $100 \mathrm{~nm}$ demonstrated superior penetration and retention in tumor tissues [36-38]. Furthermore, photothermal performance of GNR@PDA still remains unclear. Herein, we investigated PDA-coated SGNR to improve photoacoustic and photothermal performance of the core SGNR nanoparticles. First, we synthesized LGNR and SGNR that have comparable longitudinal absorption peak in NIR-II window (Figure 1a). We then coated PDA on both LGNR and SGNR to investigate both PA efficiency and photothermal conversion efficiency (Figure 1b-c). Finally, human ovarian adenocarcinoma (SKOV3) cancer cells were used to evaluate photothermal therapeutic efficiencies between LGNR (66\%), SGNR $(74 \%)$, and SGNR@PDA (95\%). Together, these size-dependent and PDA coating effects provide comprehensive insight in designing stable GNR nanoprobes for photo-mediated nanomedicine applications. 
a
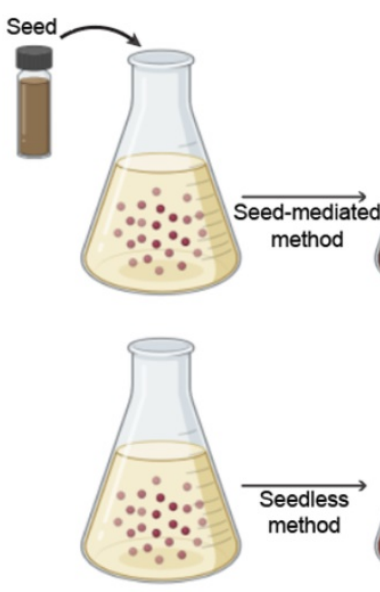
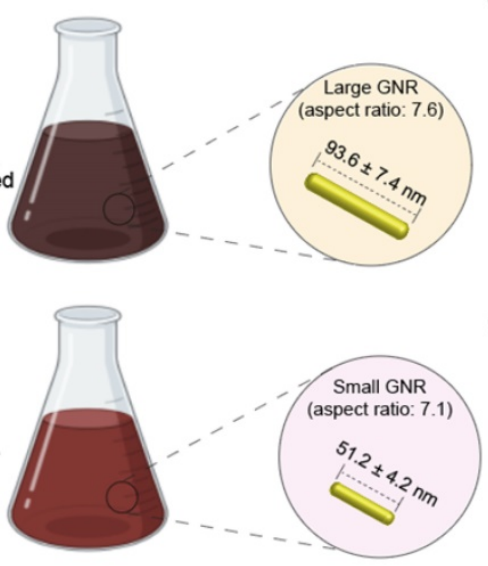

b Polydopamine coating

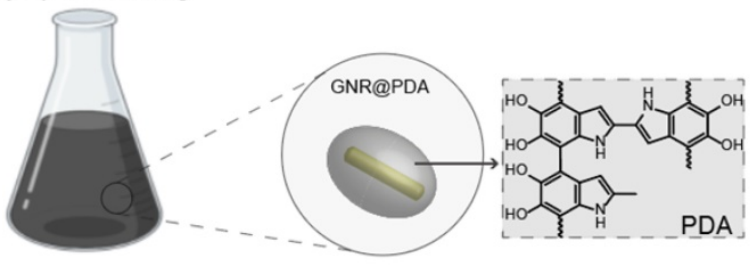

C $1064 \mathrm{~nm}$ laser

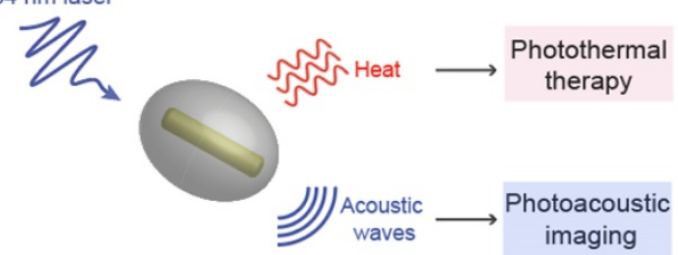

Figure 1. (a) Schematic of the synthesis of LGNRs (seed-mediated method, $93.6 \pm 7.4 \mathrm{~nm}$ in length) and SGNRs (seedless method, $51.2 \pm 4.2 \mathrm{~nm}$ in length) which have similar aspect ratio of $\sim 7$. (b) The CTAB-stabilized GNRs were replaced with HS-mPEG $2 k$ to make GNR@PDA hybrids with the PDA thickness of 25 nm. (c) PA imaging and PTT of GNR@PDAs were studied using a laser irradiation with the wavelength of $1064 \mathrm{~nm}$.

\section{Materials and Methods}

Gold(III) chloride hydrate $\left(\mathrm{HAuCl}_{4}\right)$, cetyltrimethylammonium bromide (CTAB), sodium borohydride $\left(\mathrm{NaBH}_{4}\right)$, hydroquinone, silver nitrate $\left(\mathrm{AgNO}_{3}\right)$, dopamine hydrochloride, bicine, resazurin, and McCoy's 5A medium were purchased from Sigma-Aldrich (Atlanta, GA, USA). Methoxy poly(ethylene glycol) thiol (HS-mPEG) with molecular weights $\left(\mathrm{M}_{\mathrm{w}}\right)$ of $2 \mathrm{k}, 5 \mathrm{k}, 10 \mathrm{k}$, and $20 \mathrm{k}$ were purchased from Nanocs. Dulbecco's modified Eagle's medium (DMEM) was purchased from Thermo Fisher. Calcein AM (4 mM) dissolved in DMSO was purchased from Biotium. Propidium iodide $(\geq 95 \%$, PI) was purchased from Combi-Blocks (San Diego, CA, USA). All reagents were used without further purification. Deionized water $(18.2 \mathrm{M} \Omega \cdot \mathrm{cm})$ purified with a Milli-Q Academic water purification system was used to make aqueous solutions.

\section{Synthesis of Large GNR}

We used a seed-mediated growth to synthesize regular size of GNR nanoparticles with a high aspect ratio of 7.1 [39]. A seed solution was prepared by mixing an aqueous solution of $5 \mathrm{~mL}$ of CTAB $(0.2 \mathrm{M})$ and $5 \mathrm{~mL}$ of $\mathrm{HAuCl}_{4}(0.5 \mathrm{mM})$. Then, $600 \mu \mathrm{L}$ of $\mathrm{NaBH}_{4}$ $(10 \mathrm{mM})$ was quickly injected to the aqueous solution under vigorous stirring at $1200 \mathrm{rpm}$. After $15 \mathrm{~s}$ of vigorous reaction of $\mathrm{NaBH}_{4}$, the stir bar was removed, and the seed solution was incubated in a water bath at $30{ }^{\circ} \mathrm{C}$ for $1 \mathrm{~h}$.

A growth solution was made by adding sequentially aqueous solution of $5 \mathrm{~mL}$ of CTAB $(0.2$ M), $5 \mathrm{~mL}$ of $\mathrm{HAuCl}_{4}$, and $60 \mu \mathrm{L}$ of $\mathrm{AgNO}_{3}(0.1 \mathrm{M})$. Then, $325 \mu \mathrm{L}$ of hydroquinone $(0.1 \mathrm{M})$ was injected under vigorous stirring at $1200 \mathrm{rpm}$. The color of the growth solution changed from yellow to transparent;
$165 \mu \mathrm{L}$ of the seed solution was then injected to the growth solution under vigorous stirring at $1200 \mathrm{rpm}$ for $15 \mathrm{~s}$. The solution was incubated in water bath at $30{ }^{\circ} \mathrm{C}$ for $12 \mathrm{~h}$ before being purified with centrifugation at $7,500 \mathrm{~g}$ for $10 \mathrm{~min}$. After centrifugation, CTAB-stabilized LGNRs were redispersed in $2 \mathrm{~mL}$ of deionized water.

\section{Synthesis of Ultrasmall GNRs}

We used a seedless method to synthesize ultrasmall of GNR nanoparticles with a high aspect ratio of 7.7 [20]. Briefly, $5 \mathrm{~mL}$ of CTAB $(0.2 \mathrm{M}), 4 \mathrm{~mL}$ of $\mathrm{HAuCl}_{4}(0.5 \mathrm{mM}), 30 \mu \mathrm{L}$ of $\mathrm{AgNO}_{3}(0.1 \mathrm{M}), 20 \mu \mathrm{L}$ of $\mathrm{HCl}(1.0 \mathrm{M})$, and $530 \mu \mathrm{L}$ of hydroquinone $(0.1 \mathrm{M})$ were sequentially added. The color of solution was bright yellow and stirred at $1000 \mathrm{rpm}$ for $15 \mathrm{~min}$. Finally, 20 $\mu \mathrm{L}$ of fresh $\mathrm{NaBH}_{4}(10 \mathrm{mM})$ solution (prepared within $10 \mathrm{~min}$ of use and stored on ice) was quickly injected under vigorous stirring at $1100 \mathrm{rpm}$. After $15 \mathrm{~s}$ of $\mathrm{NaBH}_{4}$ reaction, the solution was incubated in water bath at $30^{\circ} \mathrm{C}$ for $12 \mathrm{~h}$ before being purified with centrifugation at $20,000 \mathrm{~g}$ for $15 \mathrm{~min}$. After centrifugation, CTAB-stabilized SGNR was redispersed in $2 \mathrm{~mL}$ of deionized water.

\section{Surface Modification of Large and Ultrasmall GNRs}

CTAB on the surface of LGNR and SGNR was replaced with HS-mPEG $\left(\mathrm{M}_{\mathrm{w}}, 2 \mathrm{k}\right.$ Da) via ligand exchange. Both CTAB-stabilized LGNR and SGNR were dispersed in $2 \mathrm{~mL}$ of deionized water and added to $4 \mathrm{~mL}$ of HS-mPEG $(5 \mathrm{mg} / \mathrm{mL}, 1 \mathrm{mM})$ under generous stirring at $900 \mathrm{rpm}$ for $12 \mathrm{~h}$. The PEGylated LGNR was purified by centrifugation at 7,500 $\mathrm{g}$ for 10 min while the PEGylated SGNR was purified by centrifugation at 20,000 $\mathrm{g}$ for $15 \mathrm{~min}$ to remove excess of HS-mPEG in supernatant. The PEGylated LGNR 
and SGNR were redispersed in $2 \mathrm{~mL}$ of water for future use.

\section{Synthesis of PDA Coated GNRs}

The $200 \mu \mathrm{L}$ of LGNR solution was dispersed in 2 $\mathrm{mL}$ of bicine buffer $(10 \mathrm{mM}, \mathrm{pH} 8.5)$. Then, $200 \mu \mathrm{L}$ of dopamine solution $(4 \mathrm{mg} / \mathrm{mL})$ was added under vigorous stirring at $1300 \mathrm{rpm}$ for $12 \mathrm{~h}$. Similarly, 200 $\mu \mathrm{L}$ of SGNR solution was dispersed in $2 \mathrm{~mL}$ of bicine buffer (10 mM, pH 8.5) followed by $200 \mathrm{uL}$ of dopamine solution $(4 \mathrm{mg} / \mathrm{mL})$ under vigorous stirring at $1300 \mathrm{rpm}$ for $12 \mathrm{~h}$. The color of solution was dark-brown, and the resulting product was purified by centrifugation at $7,500 \mathrm{~g}$ for $10 \mathrm{~min}$. Both LGNR@PDAs and SGNR@PDAs were redispersed in water for future use.

\section{Cell Culture and Preparation}

Human ovarian adenocarcinoma (SKOV3) cells were cultured in complete McCoy's 5A medium (1.5 $\mathrm{mM}$ L-glutamine, and $2.2 \mathrm{~g} / \mathrm{L}$ sodium bicarbonate and supplemented with $10 \%$ fetal bovine serum and $1 \%$ penicillin/streptomycin). Cell cultures were incubated under $5 \% \mathrm{CO}_{2}$ at $37{ }^{\circ} \mathrm{C}$. Cultures were given at least three passages before they were used for experiments. Cells were passaged from 75 to $80 \%$ confluency using $0.25 \%$ Trypsin-EDTA. Photobleaching was used to confirm healthy and dead cells.

\section{Cytotoxicity Assay}

SKOV3 cells were seeded overnight in a 96-well plate at a concentration of 20,000 cells/well. After seeding, LGNR, SGNR, and SGNR@PDA were incubated with the SKOV3 cells at equal concentration $(10 \mu \mathrm{M})$ for $24 \mathrm{~h}$. Each well was then washed three times with cold phosphate-buffer saline (PBS) to remove the free nanoparticles. A resazurin assay was used to analyze cytotoxicity of LGNR, SGNR, and SGNR@PDA following a general protocol: After cells were incubated with resazurin for 4 hours, cell viability was calculated by measuring the subtracted background absorbance of each cell at $600 \mathrm{~nm}$ from resazurin absorbance at $570 \mathrm{~nm}$. The absorbance of experimental wells was compared to that of the controlled well including healthy and dead cells. All experiments were performed in triplicate, and the results were averaged.

\section{In Vitro PTT in NIR-II Window}

SKOV3 cells were seeded in a 96-well plate at 20,000 cells/well overnight. Each sample well was spaced out by an empty well to rule out the interference of heat transfer from the other wells during the laser irradiation. $100 \mu \mathrm{L}$ of LGNR, SGNR, and SGNR@PDA at the same concentration in $1.0 \mu \mathrm{L}$ in McCoy's 5A medium was incubated with SKOV3 cells for $24 \mathrm{~h}$. Each sample well was then exposed to a $1064 \mathrm{~nm}$ laser for $10 \mathrm{~min}$. After NIR-II laser irradiation, samples were incubated for another $12 \mathrm{~h}$, and the photothermal cytotoxicity was calculated by using a resazurin assay as described above. All experiments were done in triplicate, and the results were averaged.

\section{Cell staining with Calcein AM and Propidium lodide Dyes}

Calcein AM and Propidium Iodide (PI) were used to stain the living and dead cells before and after NIR-II laser irradiation following a general protocol. SKOV3 cells were seeded in a 24 -well plate $(50,000$ cells/well) overnight and then incubated with LGNR, SGNR, and SGNR@PDA with the same concentration of $1.0 \mu \mathrm{M}$ for $24 \mathrm{~h}$. After NIR-II laser irradiation, each sample well was incubated for $12 \mathrm{~h}$, and cells without laser irradiation was used as a control. $900 \mu \mathrm{L}$ of mixture solution of calcein AM $(2 \mu \mathrm{M})$ and PI $(6 \mu \mathrm{M})$ was added to stain the SKOV3 cells. The fluorescence images of each sample well were taken by using an EVOS FL fluorescence microscope after washing with PBS.

\section{Instrumentations}

The diluted GNR and GNR@PDA samples were dropped onto copper grids for transmission electron microscopy (TEM) measurements. TEM images were examined with a JEOL JEM 1400 Plus operating at 80 $\mathrm{kV}$. The TEM images were taken via a Gatan $4 \mathrm{k}$ digital camera. Electron-dispersive X-ray spectroscopy (EDX) samples were examined using a Thermo Fisher Talos 200X operating at $200 \mathrm{kV}$. Scanning TEM (STEM) images were examined, and EDX maps were acquired by using a Thermo Scientific software. The hydrodynamic diameter and the zeta potential of each sample were measured by using a Malvern Instrument Zetasizer ZS 90; $100 \mu \mathrm{L}$ of each sample was diluted in $900 \mu \mathrm{L}$ of distilled water. Ultravioletvisible-NIR (UV-vis-NIR) absorption spectra of each sample were measured using a PerkinElmer UV-visNIR spectrophotometer. Absorbance was read from $400 \mathrm{~nm}$ to $1350 \mathrm{~nm}$ with a step size of $3 \mathrm{~nm}$. The inductively coupled plasma mass spectrometry (ICP-MS) analysis was performed using a Thermo Scientific iCAP RQ ICP-MS in the Environmental and Complex Analysis Laboratory at UC San Diego. Samples were digested using aqua regia and prepared in $10 \mathrm{~mL}$ of $4 \% \mathrm{HNO}_{3}$. Regression analysis (R-squared) was calculated by using Microsoft Excel.

\section{Photoacoustic Imaging of LGNR, SGNR and SGNR@PDA}

A VisualSonics Vevo 2100 LAZR imaging system was used for PA imaging at $1064 \mathrm{~nm}$. Samples were 
imaged using a $21 \mathrm{MHz}$-centered LZ 250 transducer. The NIR-II laser was calibrated and optimized before the sample measurement. The specimens were positioned at a depth of $1 \mathrm{~cm}$ from the transducer. Temperature-dependent PA imaging was performed in the water bath at different temperatures (e.g., 4, 10, 20,37 , and $45^{\circ} \mathrm{C}$ ). Real-time temperature of water bath was measured using a $\mathrm{Hg}$ thermometer. Ice and hot plate were used to cool or heat the water bath. All PA data were processed using Image J software [40]. The average value and standard deviation of the PA intensity were calibrated based on the five regions of interest per tube. The R-squared and slope of PA intensity was calculated using GraphPad Prism software.

\section{Photothermal Conversion Efficiency of LGNR, SGNR, and SGNR@PDA}

Typically, $1 \mathrm{~mL}$ of LGNR, SGNR, and SGNR@PDA samples at the same concentration was placed in a quartz cuvette to measure photothermal conversion efficiency. We used a $1064 \mathrm{~nm}$ laser with the power density of $1.0 \mathrm{~W} / \mathrm{cm}^{2}$. Real-time temperature was monitored every $30 \mathrm{~s}$ by using a FLIR C5 camera. Each sample was irradiated with $1064 \mathrm{~nm}$ for $30 \mathrm{~min}$. The laser turned off after $30 \mathrm{~min}$ of irradiation, and the cooling rate was carefully recorded to calculate photothermal conversion efficiency following equation (see Supporting Information).

\section{Results and Discussion}

\section{Synthesis and characterization of SGNR@PDA}

GNRs have long been synthesized via seed-mediated growth methods [41, 42]. We used a conventional seed-mediated method to make LGNRs with $93.6 \pm 7.4 \mathrm{~nm}$ (length) $\times 12.4 \pm 1.4 \mathrm{~nm}$ (width) dimensions and an aspect ratio of 7.6 (Figure 2a) [41]. To miniaturize GNRs while maintaining high aspect ratio, the size and number of gold seeds became a limiting factor [20]. We therefore used a seedless method to make SGNRs with $51.1 \pm 4.2 \mathrm{~nm}$ (length) $\times$ $7.2 \pm 1.2 \mathrm{~nm}$ (width) dimensions and an aspect ratio of 7.1 (Figure 2b). Both LGNRs and SGNRs were stabilized with $C T A B$ and had a positive surface charge $(45.5 \pm 2.1 \mathrm{mV}$ for LGNRs, and $37.0 \pm 0.6 \mathrm{mV}$ for SGNRs). The formation of PDA coating is easily induced by auto-oxidation of dopamine under basic conditions $(\mathrm{pH}>7.5)$. Under weak alkaline conditions, dopamine undergoes successive oxidation, intramolecular cyclization, oligomerization, and then self-assembly into polymerized PDA [43]. Taking advantages of strong adhesive properties of PDA, we uniformly coated PDA on the surface of both LGNRs and SGNRs in bicine buffer ( $\mathrm{pH}$ 8.5). TEM images show monodispersed LGNR@PDA and SGNR@PDA nanohybrids with same $25 \mathrm{~nm}$ PDA coating thickness (Figure 2c-d). The coating thickness was readily tunable from $5 \mathrm{~nm}$ to $50 \mathrm{~nm}$ by adjusting the amount of dopamine feed $(4 \mathrm{mg} / \mathrm{mL})$ in the reactions (Figure S1). EDX mapping and high-angle annular dark-field imaging (HAADF) confirmed the core - shell nanostructures of SGNR-polydopamine hybrids composed of $\mathrm{Au}, \mathrm{C}, \mathrm{N}$, and $\mathrm{O}$ (Figure 2e and Figure S2). Furthermore, EDX line scanning indicated that SGNR nanoparticles were covered by carbon-the main component of PDA (Figure 2f). Adding dopamine directly to CTAB-stabilized LGNRs and SGNRs led to particle aggregation due to strong electrostatic interactions between positively charged GNRs and negatively charged dopamine. Therefore, we replaced CTAB with a methoxy PEG thiol of $\mathrm{M}_{\mathrm{w}}$ 2k Da (HS-mPEG) to accommodate stable PDA coating (Figure S3). Using strong Au-thiol bonding formation [44], we successfully exchanged the surfaces of both LGNRs and SGNRs with HS-mPEG, where the zeta potential of LGNRs and SGNRs decreased to $8.3 \pm 2.6 \mathrm{mV}$ and $4.6 \pm 0.6 \mathrm{mV}$, respectively (Figure 2g). PEGylated LGNRs and SGNRs carry more negative charges after PDA coating due to the phenolic hydroxyl groups on PDA. Histogram of SGNR@PDA and LGNR@PDA sizes as measured by TEM shows that the size of LGNR@PDA nanohybrids is two-fold larger than that of SGNR@PDA nanohybrids (Figure 2h).

\section{Photoacoustic performance of SGNR@PDAs}

When LSPR nanoparticles are in solution, light is mainly absorbed by the nanoparticles rather than the solvent. The plasmonic SGNR nanoparticles convert absorbed light energy into heat generating acoustic energy into the surrounding medium. Both heat and stress leak out from the nanoparticles during nanosecond-pulsed laser irradiation due to high thermal conductivity of gold and nanometric volume of the particles [45]. Therefore, PA signal is determined by optical absorption of nanoparticles as well as heat transfer rate from gold to water [46]. UVvis-NIR absorption spectra showed that LGNR had two absorption peaks at $504 \mathrm{~nm}$ and $1076 \mathrm{~nm}$ that correspond to the transverse and longitudinal LSPR, respectively. Likewise, SGNR exhibited two absorption peaks at $512 \mathrm{~nm}$ and $1064 \mathrm{~nm}$, validating that GNR aspect ratio determines its LSPR absorption peaks (Figure 3a and Figure S5) [47]. The longitudinal peaks of both GNRs were significantly redshifted when PDA was coated due to the increased refractive index $(n)$ from the surrounding medium $\left(n_{P D A} \approx 1.7\right.$, and $n_{\text {water }} \approx 1.3$ ) [48]. 

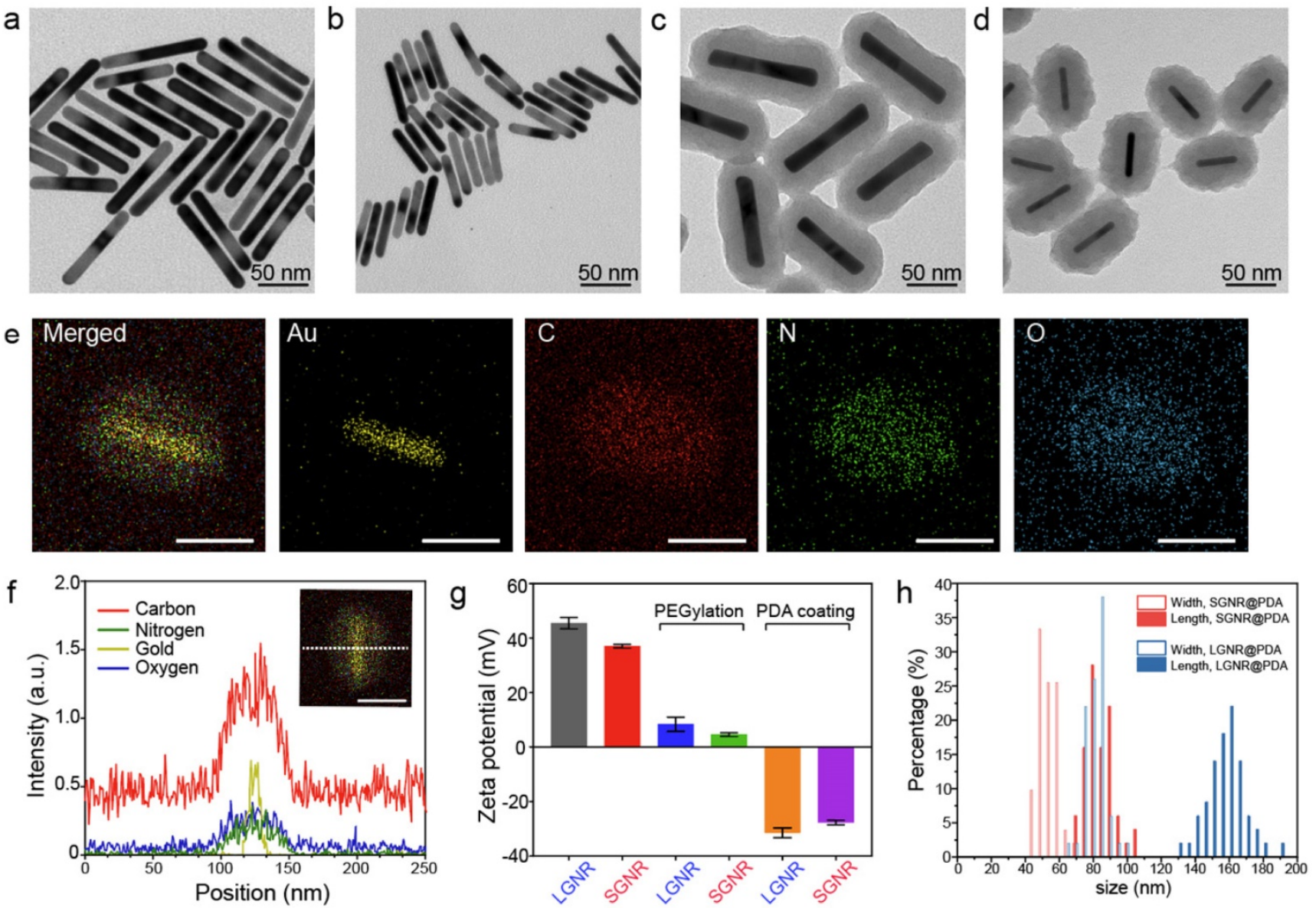

Figure 2. PDA-coated large and ultrasmall GNRs. TEM images of (a) LGNRs, (b) SGNRs, (c) LGNR@PDA, and (d) SGNR@PDA. Same PDA thickness about 25 nm was coated on both LGNRs and SGNRs. (e) EDX mapping of SGNR@PDAs shows the core-shell nanostructure of SGNR-polydopamine nanohybrids. The scale bar represents 50 nm. (f) EDX line scanning of SGNR@PDAs. The inset image indicates the location of SGNR@PDAs that were used to analyze the signal intensity of Au, C, N, and O. (g) Zeta potential of LGNR and SGNR before and after PEGylation and PDA coating. Zeta potential of CTAB-stabilized GNRs decreased after ligand exchange, and the surface charge of PEGylated GNRs further decreased when coated with negatively charged PDA. The error bar represents standard deviation of five measurements. (h) Histogram of SGNR@PDA and LGNR@PDA indicates that the length of LGNR@PDA is 2-fold larger than that of SGNR@PDA. Fifty nanoparticles were used to calibrate the width and length of the GNR@PDA nanohybrids.

We illuminated optical density (OD)-matched GNR solutions at $1064 \mathrm{~nm}$ to measure PA performance of LGNR and SGNR. Consistent with a previous study [20], SGNR (OD $\approx 1$ at $1064 \mathrm{~nm}$ ) showed 3-fold higher PA intensity than LGNR (OD $\approx$ 1 at $1064 \mathrm{~nm}$ ) because of its higher surface-to-volume ratio that can improve heat transfer (Figure 3b) [47]. We then used inductively coupled plasma mass spectrometry (ICP-MS) to match each GNR and GNR@PDA concentrations based on the number of $\mathrm{Au}$ ions in each sample (Figure S4). As a result, optical extinctions of both LGNR@PDA and SGNR@PDA were higher than pristine LGNR and SGNR due to the absorbance of PDA coating and the increased size of GNR-melanin hybrid nanoparticles. The increase in absorption intensity is important because PA signal is directly a function of the optical and thermoelastic properties of nanoparticles according to the thermoelastic expansion model (equation 1) [3].

$$
\rho_{o}=\left(\frac{\beta c^{2}}{C_{P}}\right) \mu_{\alpha} F
$$

where, $\rho_{o}, \beta, c, \mathrm{C}_{P}, \mu_{\alpha}$, and $F$ are pressure gradient, thermoelastic expansion coefficient, speed of sound in the medium, specific heat capacity, absorption coefficient, and laser fluence, respectively.

PDA coating further improved PA performance of both LGNR and SGNR (Figure 3b). LGNR@PDA and SGNR@PDA showed three-fold higher PA signals than their corresponding pristine LGNR and SGNR. Moreover, SGNR@PDA showed 3-fold higher PA intensity than LGNR@PDA due to the inherent PA enhancement from the core SGNRs. This significant improvement in PA performance of GNR@PDAs can be explained by three parameters: optical absorption, cross-sectional area, and thermal confinement. First, the melanin-like PDA coating improved optical absorption of GNR as observed in Figure 3a. Consequently, GNR@PDAs can absorb more photons than pristine GNR and therefore generate more 
acoustic energy. Second, the cross-sectional area of GNR@PDA, as measured by electron microscopy, became larger because the PDA coating itself can absorb light. Lastly, thermal confinement obtained from the PDA shell leads to higher PA signal. The heat capacity of water $\left(\mathrm{C}_{\text {water }} \approx 4.2\right)$ is 2.5 times higher than that of PDA $\left(\mathrm{C}_{\mathrm{PDA}} \approx 1.6\right)$ which means that the GNR surrounded by water requires higher energy to heat versus GNR surrounded by a PDA shell [49]. Furthermore, the thermal conductivity of water $\left(K_{\text {water }}\right.$ $\approx 0.59)$ is 4 times larger than that of PDA $\left(K_{\mathrm{PDA}} \approx 0.13\right)$ indicating that heat can readily accumulate in GNR@PDAs due to the reduced thermal conductance [49]. Collectively, the PDA coating gave both LGNR and SGNR higher thermoelastic expansions than GNR alone to significantly increase PA signals.

PA signal generation is also dependent upon other factors including particle concentration and thermal expansion of surrounding solvent. As optical extinction of nanoparticles is linearly proportional to particle concentration, a linear increase in PA signal was observed with elevated concentrations (Figure 3c). Importantly, SGNR@PDA at the concentration of $0.2 \mu \mathrm{M}$ still showed high PA signal which was 15 -fold higher than that of SGNR (Figure 3c). The thermal expansion coefficient is an inherent material-based property, and PA signal is generated by a sum of the thermal expansion of the nanoparticle and its surrounding solvent. During nanosecond-pulsed laser irradiation, heat is not only confined within the nanoparticles but also diffused into the solvent, leading to a shell-like layer of the solvent around the nanoparticles with the increased temperature [3]. To study the thermal expansion of GNR@PDAs, we measured their PA signals in different temperatures: $4,10,20,37$, and $45^{\circ} \mathrm{C}$. Since water was the most dense at $3.98{ }^{\circ} \mathrm{C}$, thermal expansion of water became very low [46]. That is, PA signal at $3.98{ }^{\circ} \mathrm{C}$ is mainly due to the thermal expansion of nanoparticles. LGNR, SGNR, LGNR@PDA, and SGNR@PDA nanoparticles showed the lowest PA signals at $4{ }^{\circ} \mathrm{C}$ indicating that the thermal expansion of the solvent around the nanoparticles is involved in PA signal generation (Figure 3d). Importantly, the PA signal from SGNR@PDA at $10^{\circ} \mathrm{C}$ significantly increased ( $\sim 3$ times higher than that at $4{ }^{\circ} \mathrm{C}$ ) while PA signal of SGNR alone remained low. This result indicates that thermal confinement of PDA shell contributed to thermal expansion of SGNR@PDA (Figure 3d).

The PA signals of LGNR, SGNR, LGNR@PDA, and SGNR@PDA linearly increased with increased temperature of the solvent. More importantly, the Grüneisen parameter of LGNR, SGNR, LGNR@GNR, or SGNR@PDA was measured based on the slope of temperature versus PA intensity because the
Grüneisen parameter is a function of the thermal expansion coefficient and specific heat capacity, which are temperature-dependent $[3,46]$. The Grüneisen parameter (dimensionless) of SGNR@PDA (0.066) was 2.4-fold higher than that of SGNR (0.027). Likewise, Grüneisen parameters of LGNR@PDA (0.038) was 1.7-fold higher than that of LGNR (0.022). These results confirm that the PDA coating can improve the thermal conversion efficiency of core GNR nanoparticles.

\section{Photothermal performance of SGNR@PDAs}

Next, we examined the photothermal performance of LGNR, SGNR, and SGNR@PDA in NIR-II using a laser of $1064 \mathrm{~nm}$ for excitation. The skin-tolerance threshold set by the America Standards Institute is MPE of $1.0 \mathrm{~W} / \mathrm{cm}^{2}$ for $1064 \mathrm{~nm}$ laser (ANSI Z136.1-2007); we therefore chose a $1064 \mathrm{~nm}$ laser power of $1.0 \mathrm{~W} / \mathrm{cm}^{2}$ to evaluate the photothermal properties of GNR-melanin nanohybrids. Figure 4a shows that the temperature of LGNR, SGNR, SGNR@PDA at the same concentration of $10 \mu \mathrm{g} / \mathrm{mL}$ increased to $46.5^{\circ} \mathrm{C}, 49.8^{\circ} \mathrm{C}$ and $57.3^{\circ} \mathrm{C}$, respectively within $10 \mathrm{~min}$; temperature change of water was negligible. Photothermal conversion efficiency is the capacity of nanoparticle to convert laser energy into heat. The laser turned off after $30 \mathrm{~min}$ of irradiation, and the cooling rate was recorded every 30 seconds to measure heat transferring from the nanoparticles to surroundings. Compared to other agents such as GNR@Ag (28.8\%), Au@Metal-Organic Framework $(30.2 \%)$ [50, 51], the SGNR@PDA showed high photothermal conversion efficiency (40\%) at $1064 \mathrm{~nm}$. This value was also higher than SGNR $(30 \%)$ and LGNR (27\%) (Figure 4b). Furthermore, the photothermal conversion efficiency of PDA nanoparticle was $18 \%$ at $1064 \mathrm{~nm}$ indicating that GNR-melanin nanohybrids can offer outstanding photothermal performance (Figure S6). Superior photothermal efficiency of SGNR@PDA results from a higher optical absorption, large surface area of PDA, and improved thermal conversion efficiency (Grüneisen parameter) due to the thermal confinement of the PDA shell. Likewise, photothermal conversion efficiency of LGNR@PDA was $4 \%$ higher than LGNR (Figure S7). The thermal images also showed SGNR@PDA rapidly increased temperature to $50{ }^{\circ} \mathrm{C}$ within one minute compared to SGNR and LGNR, which required three to four minutes to achieve a similar temperature (Figure 4c).

SGNR@PDAs showed excellent conversion stability in four successive cycles confirming its good photostability in the NIR-II window (Figure 4d). In addition, SGNR@PDAs did not show any disassembly of PDA coating or morphology changes after four 
successive cycles of laser irradiation (Figure S9). The PDA coating can improve photothermal stability of GNR during laser irradiation because gold atoms on the surface of GNR@PDAs are tightly immobilized because of the highly crosslinked PDA shell and $\Pi$-stacking structure [32]. Therefore, the PDA shell serves as physical barrier while the atomic rearrangement in the GNR requires more energy. On the other hand, pristine GNRs alone cannot afford extensive heating [32]. We further studied the photothermal property of SGNR@PDA in different particle concentrations and power densities. The temperature changes $(\Delta \mathrm{T})$ showed that the temperature increase was linearly proportional to the particle concentration. For example, $\Delta \mathrm{T}$ of
SGNR@PDA was $19.1{ }^{\circ} \mathrm{C}, 22.2^{\circ} \mathrm{C}, 24.4^{\circ} \mathrm{C}$, and $25.5^{\circ} \mathrm{C}$ for concentrations of $4,6,8$, and $10 \mu \mathrm{g} / \mathrm{mL}$ after $7 \mathrm{~min}$ of NIR-II laser irradiation at the same power density of $1.0 \mathrm{~W} / \mathrm{cm}^{2}$ (Figure $4 \mathrm{e}$ ).

The $\Delta \mathrm{T}$ of SGNR@PDA also corresponded to the laser power density. For example, the temperature of SGNR@PDA increased to $20.8^{\circ} \mathrm{C}, 25.4^{\circ} \mathrm{C}, 27.5^{\circ} \mathrm{C}$, and $36.7^{\circ} \mathrm{C}$ at the laser power densities of $0.9 \mathrm{~W} / \mathrm{cm}^{2}, 1.1$ $\mathrm{W} / \mathrm{cm}^{2}, 1.3 \mathrm{~W} / \mathrm{cm}^{2}, 1.5 \mathrm{~W} / \mathrm{cm}^{2}$ at the fixed concentration of $10 \mu \mathrm{g} / \mathrm{mL}$ (Figure $4 \mathbf{f}$ ). Collectively, these findings corroborated distinctive thermophilic characteristics and high photothermal conversion efficiency of SGNR@PDA highlighting its potential use in therapeutic agent for cancer treatment. a

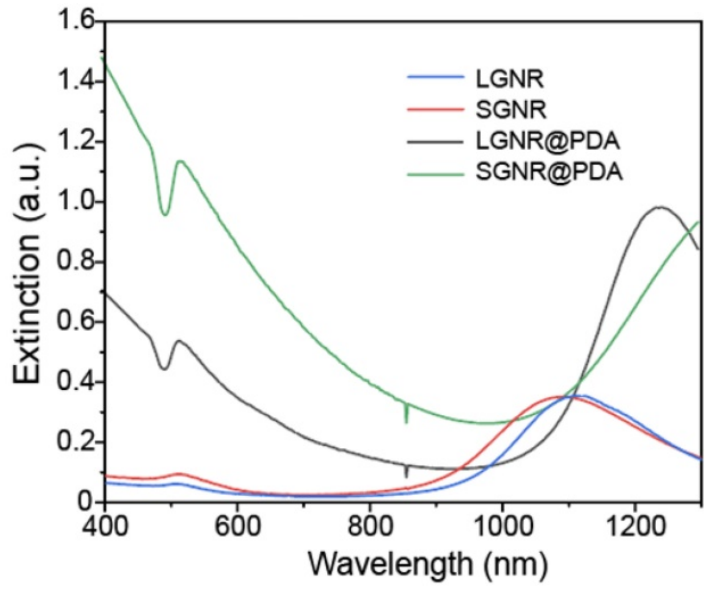

C

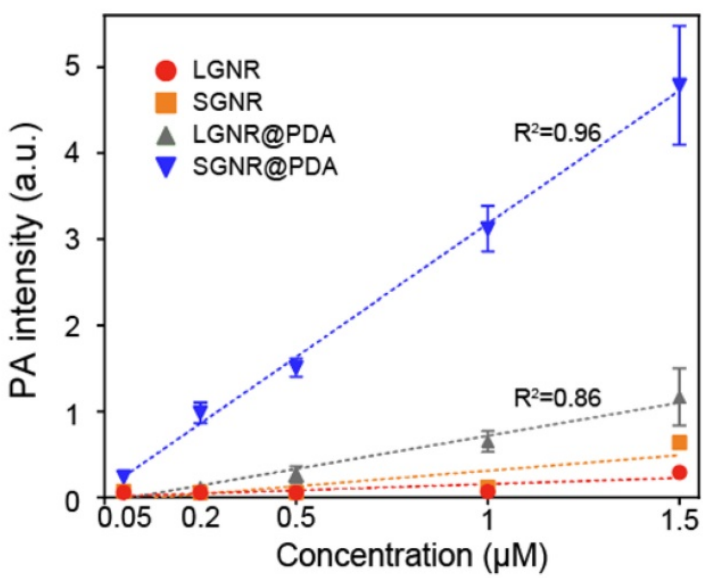

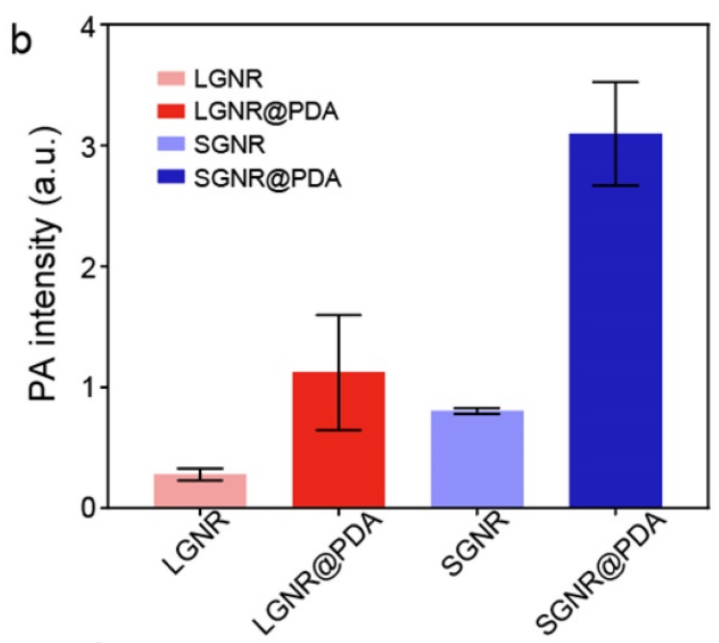

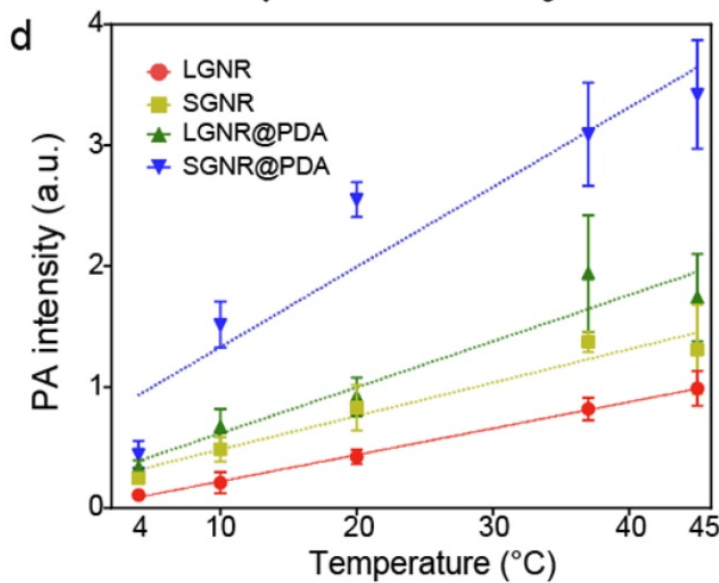

Figure 3.PA imaging of GNR@PDAs (a) UV-vis-NIR absorption spectra of LGNR, SGNR, LGNR@PDA, and SGNR@PDA. Longitudinal absorption peaks of LGNR and SGNR redshifted after PDA coating due to the increased refractive index. Molar concentrations of pristine GNR and GNR@PDA are the same. (b) PA intensity of LGNR, LGNR@PDA, SGNR, and SGNR@PDA under NIR-II (1064 nm) laser irradiation. PDA coating improved PA performance of core GNR particles. (c) PA intensity of LGNR, SGNR, LGNR@PDA, and SGNR@PDA with the elevated sample concentration under NIR-II (1064 nm) laser irradiation. SGNR@PDA showed 4-fold higher PA signal generation than pristine SGNR. (d) PA intensity of LGNR, SGNR, LGNR@PDA, and SGNR@PDA measured in different temperature (e.g., 4, 10, 20, 37, and 45 $\left.{ }^{\circ} \mathrm{C}\right)$. Slopes of temperature versus PA intensity of LGNR, SGNR, LGNR@PDA, and SGNR@PDA are 0.022, 0.027, 0.038, and 0.066, respectively. The error bars represent the standard deviation of five regions of interest. 

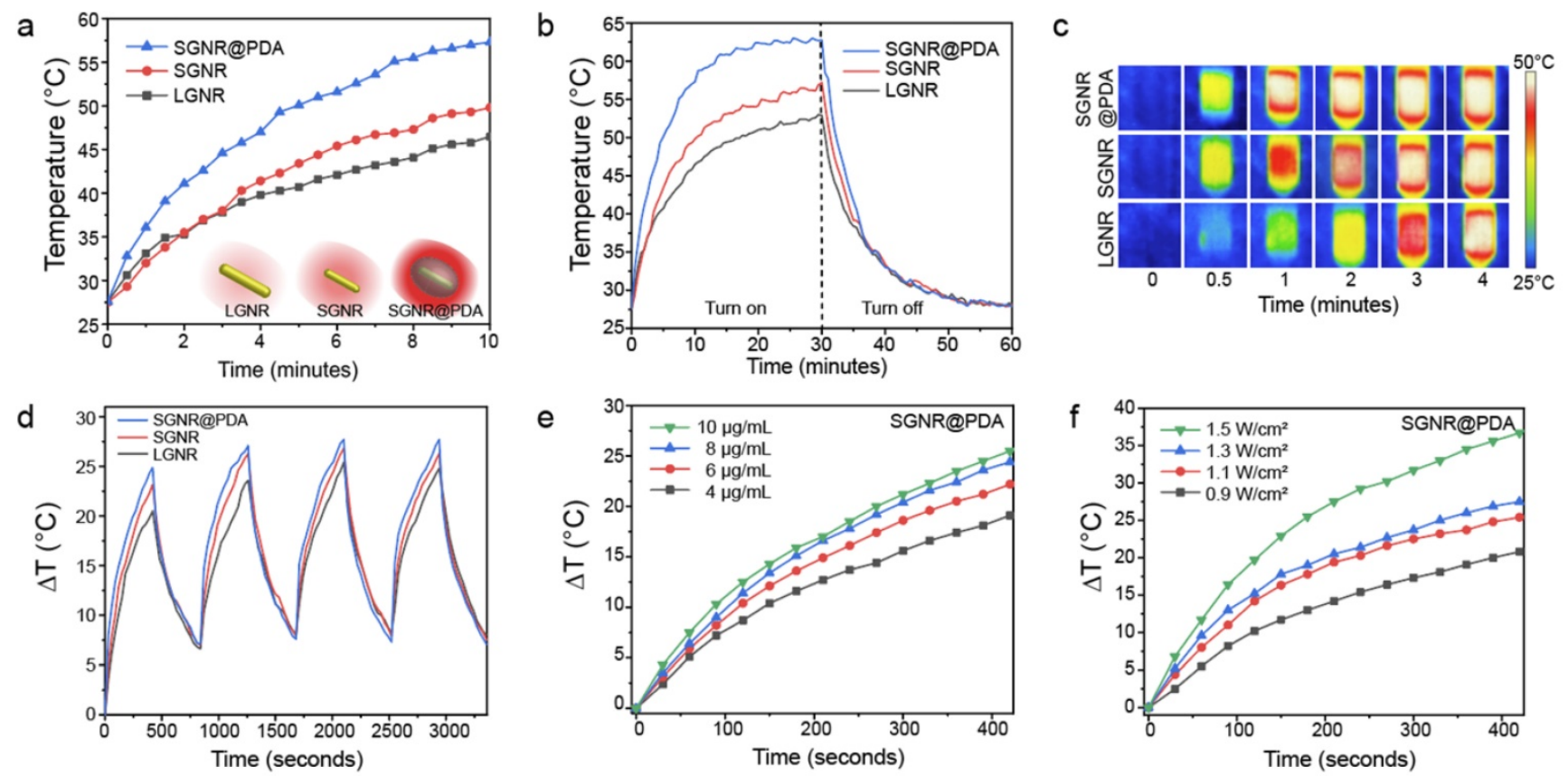

Figure 4. (a) Schematic illustration of the photothermal performance of LGNR, SGNR, and SGNR@PDA triggered by NIR-II (1064 nm) laser. (b) Temperature curves of LGNR, SGNR, and SGNR@PDA for $1 \mathrm{~h}$ under NIR-II (1064 nm) laser irradiation, showing that the temperature of SGNR@PDA largely increased compared to SGNR and LGNR. (c) Thermal images of LGNR, SGNR, and SGNR@PDA irradiated at 1064 nm. (d) Temperature changes of LGNR, SGNR, and SGNR@PDA under repeated “on and off” laser irradiation for four times cycle. (e) Temperature changes of SGNR@PDA with the elevated concentrations (e.g., 4, 6, 8, and 10 $\mu$ g/mL) for 7 min irradiation. (f) Temperature changes of SGNR@PDA at different power densities $\left(0.9,1.1,1.3\right.$, and $\left.1.5 \mathrm{~W} / \mathrm{cm}^{2}\right)$ for 7 min irradiation. Temperature curves of SGNR and LGNR are shown in Figure S8.

\section{Photothermal treatment of SGNR@PDA in vitro}

We investigated the PTT efficacy of the SGNR@PDA at the NIR-II window in vitro. SKOV3 cancer cells were incubated with LGNR, SGNR, or SGNR@PDA with different concentrations from 0.2 $\mu \mathrm{M}$ to $1.2 \mu \mathrm{M}$ for $24 \mathrm{~h}$. After co-incubation for $24 \mathrm{~h}$, the cell viabilities of SKOV3 were greater than $90 \%$ indicating that both PEGylated GNRs and GNR@PDAs had negligible cytotoxicity (Figure 5a). We then studied photothermal ablation toward SKOV3 cells when they were incubated with LGNR, SGNR, or SGNR@PDA at the same concentration (1.0 $\mu \mathrm{M})$. Importantly, SGNR@PDA showed effective cell ablation (95\%) after $10 \mathrm{~min}$ of laser irradiation at 1064 nm while cell ablations from LGNR and SGNR were $66 \%$ and $74 \%$, respectively (Figure $5 \mathbf{b}$ ). SKOV3 cells without nanoparticles retained high cell viability before $(100 \%)$ and after $(99 \%)$ laser irradiation validating that LGNR, SGNR, and SGNR@PDA are the main factors to elicit photothermal ablation of SKOV3 cells (Figure 5b).

The photothermal treatment from LGNR, SGNR, and SGNR@PDA was further studied and visualized via live-dead cell fluorescent stanning. Calcein-AM (green fluorescence for live cells) and PI (red fluorescence of dead cells) were used in these experiments. Strong green fluorescence was observed when SKOV3 cells were incubated with LGNR,
SGNR, or SGNR@PDA before NIR-II laser irradiation (Figure 5c). After $10 \mathrm{~min}$ of NIR-II laser irradiation, SKOV3 cells treated with LGNR, SGNR, or SGNR@PDA showed red fluorescence. SKOV3 cells incubated with SGNR@PDA showed strong red fluorescence, which corresponds to quantitative data: The cell ablation of SGNR@PDA was 95\% (Figure $\mathbf{5 b}-\mathbf{c})$. The SKOV3 cells alone were irradiated with NIR-II laser for $10 \mathrm{~min}$ to validate the lack of involvement of the laser toward the photothermal ablation; strong green fluorescence was observed before and after laser irradiation (Figure 5c). These results indicated that the NIR-II laser alone could not cause cell death, and photothermal performance of SGNR significantly improved when PDA was coating due to higher temperature induced from the PDA shell. Finally, the colloidal stability of SGNR@PDA was investigated in different media (e.g., $10 \mathrm{mM}, \mathrm{HCl}$, $10 \mathrm{mM} \mathrm{NaOH}, 10 \mathrm{mM} \mathrm{NaCl}$, DMEM with $10 \%$ fetal bovine serum, and DMEM with the human serum of $10 \%)$. DLS data showed that SGNR@PDA maintained its GNR-melanin assemblies and showed negligible aggregation under those conditions (PDI $\sim 0.1$ ) (Figure S10). These results indicate that PDA coating is highly intact and stable under biological conditions. Therefore, our PDA coating strategy can improve photothermal performance over the SGNR probe with excellent colloidal stability. 

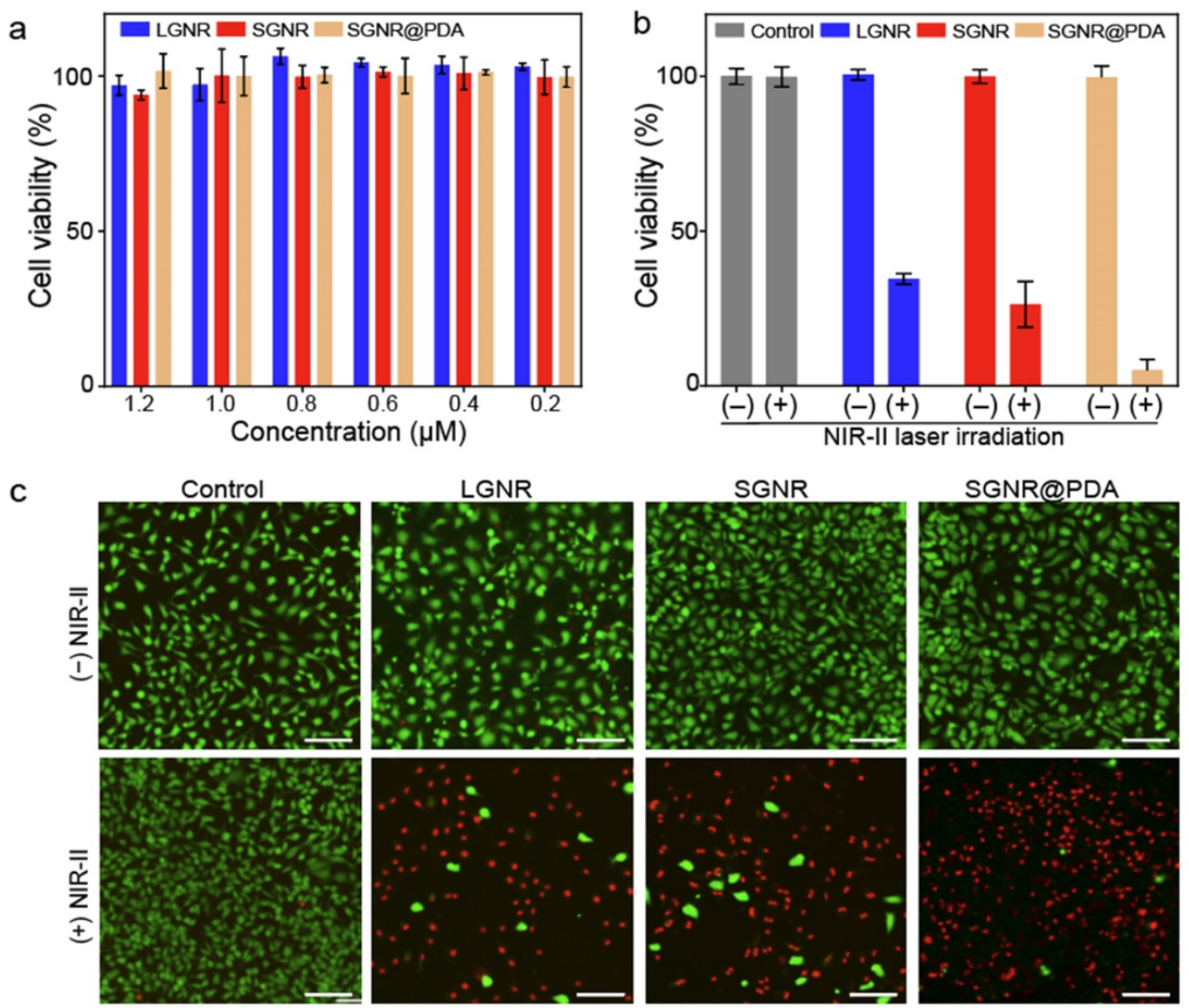

Figure 5. Photothermal performance of SGNR@PDA on SKOV3 cancer cell. (a) Cell viability of SKOV3 cells after co-incubated for $24 \mathrm{~h}$ with LGNR, SGNR, and SGNR@PDA at different concentrations. (b) Cell viability of SKOV3 cells before and after photothermal treatment $\left(1.0 \mathrm{~W} / \mathrm{cm}^{2} \mathrm{for} 1064 \mathrm{~nm}, 10 \mathrm{~min}\right)$ at the same concentration of LGNR, SGNR, and SGNR@PDA (1.0 $\mu \mathrm{M})$, indicating that SGNR@PDAs showed high photothermal-induced cell ablation compared to LGNR and SGNR. SKOV3 cells without injecting nanoparticles (referred as Control) were exposed to the laser, showing that there was no involvement of cell medium to kill the SKOV3 cells. The error bar represents the standard deviation of three measurements. (c) Fluorescence images of Control, LGNR, SGNR, and SGNR@PDA stained with Calcein AM and PI before and after laser irradiation at $1064 \mathrm{~nm}\left(1.0 \mathrm{~W} / \mathrm{cm}^{2}\right.$ for $\left.1064 \mathrm{~nm}, 10 \mathrm{~min}\right)$. Photobleaching was used to confirm healthy and dead SKOV3 cells (Figure S1 1). The scale bar indicates $100 \mu \mathrm{m}$.

Although proof-of-concept, this work invites more studies to improve the viability of melanin-GNRs for anticancer therapy in vivo. One limitation of this work is lack of tumor targeting in vivo. In a mouse model, the photothermal performance of LGNR, SGNR, and SGNR@PDA species could be less efficient due to tissue thickness, light scattering, and absorbance from the tissue. More importantly, we do show higher photothermal performance of SGNR@PDA than SGNR due to improved optical absorption, and higher thermal conversion efficiency due to the thermal confinement of PDA shell. Future work will include photothermal treatment of SGNR@PDA in mouse model for clinical application of NIR-II PTT.

\section{Conclusion}

Plasmonic nanomaterials, especially GNRs, hold great promise in photo-mediated applications due to their tunable and high absorption efficiency. However, anisotropic nanostructures under the laser irradiation become unstable, losing their unique optical properties. In this study, we synthesized both large GNR and ultrasmall GNR to investigate 'size-effect' and further synthesized GNR-melanin hybrids (i.e., GNR@PDA) to study 'PDA coating effect' altogether. SGNRs showed higher PA performance over large GNR due to high surface-to-volume ratio. Furthermore, SGNR@PDA showed three-fold higher PA signal than core SGNR nanoparticles and $10 \%$ higher photothermal efficiency over SGNR due to improved optical absorption and 2.4-fold higher thermal conversion efficiency. These SGNR@PDA nanohybrids also elicited effective photothermal ablation of SKOV3 cancer cells (95\%) which was significantly higher than LGNR $(66 \%)$ and SGNR (74\%). These findings indicate high potential use of the PDA coating strategy in developing photo-mediated biomedicines in NIR-II window and expand our understanding photothermal conversion mechanism occurring in hybrid nanostructures. 


\section{Abbreviations}

PA: photoacoustic; PTT: photothermal therapy; NIR-II: second near-infrared; GNRs: gold nanorods; PDA: polydopamine; GNR@PDAs: polydopaminecoated gold nanorods; UV-vis-NIR: ultravioletvisible-NIR; HAADF: High-angle annular dark-field imaging; ICP-MS: inductively coupled plasma mass spectrometry; LSPR: localized surface plasmonic resonance; SKOV3: human ovarian adenocarcinoma; DLS: dynamic light scattering; PDI: polydispersity index; EDX: Electron-dispersive X-ray spectroscopy; TEM: transmission electron microscopy.

\section{Supplementary Material}

Supplementary figures.

http://www.ntno.org/v06p0079s1.pdf

\section{Acknowledgments}

The authors acknowledge the National Science Foundation under award 1937674 and 1845683 . We also thank National Institute of Health under awards DP2 HL137187. The authors also acknowledge infrastructure support under awards S10 OD021821 and S10 OD023555. MNC and RMB acknowledge fellowship support under T32 CA153915. Transmission electron micrographs were taken in the Cellular and Molecular Medicine Electron Microscopy Core Facility, which is supported by National Institutes of Health award number S10 OD 023527. This work was performed in part at the San Diego Nanotechnology Infrastructure (SDNI) of University of California San Diego, a member of the National Nanotechnology Coordinated Infrastructure (NNCI), which is supported by the National Science Foundation (Grant ECCS-1542148).

\section{Competing Interests}

The authors have declared that no competing interest exists.

\section{References}

1. Zhao P, Jin Z, Chen Q, Yang T, Chen D, Meng J, et al. Local generation of hydrogen for enhanced photothermal therapy. Nat Commun. 2018; 9: 1-12

2. Weber J, Beard PC, Bohndiek SE. Contrast agents for molecular photoacoustic imaging. Nat Methods. 2016; 13: 639-50.

3. Mantri Y, Jokerst JV. Engineering plasmonic nanoparticles for enhanced photoacoustic imaging. ACS Nano. 2020; 14: 9408-22.

4. Li Q, Li S, He S, Chen W, Cheng P, Zhang Y, et al. An activatable polymeric reporter for near-infrared fluorescent and photoacoustic imaging of invasive cancer. Angew Chem. 2020; 132: 7084-9.

5. Zhou J, Jokerst JV. Photoacoustic imaging with fiber optic technology: A review. Photoacoustics. 2020: 100211.

6. Borum RM, Jokerst JV. Hybridizing clinical translatability with enzyme-free DNA signal amplifiers: recent advances in nucleic acid detection and imaging. Biomater Sci. 2020

7. Jeevarathinam AS, Lemaster JE, Chen F, Zhao E, Jokerst JV. Photoacoustic imaging quantifies drug release from nanocarriers via redox chemistry of dyelabeled cargo. Angew Chem Inter Edi. 2020; 59: 4678-83.

8. Liu S, Pan X, Liu H. Two-dimensional nanomaterials for photothermal therapy. Angew Chem. 2020; 132: 5943-53
9. Jung HS, Verwilst P, Sharma A, Shin J, Sessler JL, Kim JS. Organic molecule-based photothermal agents: an expanding photothermal therapy universe. Chem Soc Rev. 2018; 47: 2280-97.

10. Day ES, Morton JG, West JL. Nanoparticles for thermal cancer therapy. J Biomech Eng. 2009; 131.

11. Zhou J, Jiang Y, Hou S, Upputuri PK, Wu D, Li J, et al. Compact plasmonic blackbody for cancer theranosis in the near-infrared II window. ACS Nano. 2018; 12: 2643-51.

12. Jiang Y, Zhao X, Huang J, Li J, Upputuri PK, Sun H, et al. Transformable hybrid semiconducting polymer nanozyme for second near-infrared photothermal ferrotherapy. Nat Commun. 2020; 11: 1-13.

13. Li J, Yu X, Jiang Y, He S, Zhang Y, Luo Y, et al. Second near-infrared photothermal semiconducting polymer nanoadjuvant for enhanced cancer immunotherapy. Adv Mater. 2021; 33: 2003458.

14. Xu M, Yim W, Zhou J, Zhou J, Jin Z, Moore C, et al. The application of organic nanomaterials for bioimaging, drug delivery, and therapy: spanning various domains. IEEE Nanotechnol Mag. 2021.

15. Chitgupi U, Nyayapathi N, Kim J, Wang D, Sun B, Li C, et al. Surfactantstripped micelles for NIR-II photoacoustic imaging through $12 \mathrm{~cm}$ of breast tissue and whole human breasts. Adv Mater. 2019; 31: 1902279.

16. $\mathrm{Xu} \mathrm{C}, \mathrm{Pu} \mathrm{K}$. Second near-infrared photothermal materials for combinational nanotheranostics. Chem Soc Rev. 2021.

17. Jiang Y, Huang J, Xu C, Pu K. Activatable polymer nanoagonist for second near-infrared photothermal immunotherapy of cancer. Nat Commun. 2021; 12: 1-14.

18. Chang S-S, Shih C-W, Chen C-D, Lai W-C, Wang CC. The shape transition of gold nanorods. Langmuir. 1999; 15: 701-9.

19. Link S, Wang ZL, El-Sayed MA. How does a gold nanorod melt? J Phys Chem B. 2000; 104: 7867-70

20. Chen Y-S, Zhao Y, Yoon SJ, Gambhir SS, Emelianov S. Miniature gold nanorods for photoacoustic molecular imaging in the second near-infrared optical window. Nat Nanotech. 2019; 14: 465-72.

21. Kaneti YV, Chen C, Liu M, Wang X, Yang JL, Taylor RA, et al. Carbon-coated gold nanorods: a facile route to biocompatible materials for photothermal applications. ACS Appl Mater Interfaces. 2015; 7: 25658-68.

22. Zhao Y-Q, Sun Y, Zhang Y, Ding X, Zhao N, Yu B, et al. Well-defined gold nanorod/polymer hybrid coating with inherent antifouling and photothermal bactericidal properties for treating an infected hernia. ACS Nano. 2020; 14: 2265-75.

23. Zhang Z, Wang L, Wang J, Jiang X, Li X, Hu Z, et al. Mesoporous silica-coated gold nanorods as a light-mediated multifunctional theranostic platform for cancer treatment. Adv Mater. 2012; 24: 1418-23.

24. Zhou J, Lin Z, Penna M, Pan S, Ju Y, Li S, et al. Particle engineering enabled by polyphenol-mediated supramolecular networks. Nat Commun. 2020; 11: 1-8.

25. Del Caño R, Gisbert-González JM, González-Rodríguez J, Sánchez-Obrero G, Madueño $R$, Blázquez $M$, et al. Effective replacement of cetyltrimethylammonium bromide (CTAB) by mercaptoalkanoic acids on gold nanorod (AuNR) surfaces in aqueous solutions. Nanoscale. 2020; 12: 658-68.

26. Shi X, Perry HL, Wilton-Ely JD. Strategies for the functionalisation of gold nanorods to reduce toxicity and aid clinical translation. Nanotheranostics. 2021; 5: 155.

27. Wang L, Li D, Hao Y, Niu M, Hu Y, Zhao H, et al. Gold nanorod-based poly (lactic-co-glycolic acid) with manganese dioxide core-shell structured multifunctional nanoplatform for cancer theranostic applications. Int $\mathrm{J}$ Nanomed. 2017; 12: 3059.

28. Lemaster JE, Wang Z, Hariri A, Chen F, Hu Z, Huang Y, et al. Gadolinium doping enhances the photoacoustic signal of synthetic melanin nanoparticles: a dual modality contrast agent for stem cell imaging. Chem Mater. 2018; 31: $251-9$

29. Siwicka ZE, Son FA, Battistella C, Moore MH, Korpanty J, McCallum NC, et al. Synthetic porous melanin. J Am Chem Soc. 2021; 143: 3094-103.

30. Wu D, Zhou I, Creyer MN, Yim W, Chen Z, Messersmith PB, et al Phenolic-enabled nanotechnology: versatile particle engineering for biomedicine. Chem Soc Rev. 2021

31. Lee H, Dellatore SM, Miller WM, Messersmith PB. Mussel-inspired surface chemistry for multifunctional coatings. Science. 2007; 318: 426-30.

32. Yim W, Zhou J, Mantri Y, Creyer MN, Moore CA, Jokerst JV. Gold nanorodmelanin hybrids for enhanced and prolonged photoacoustic imaging in the near-infrared-II window. ACS Appl Mater Interfaces. 2021.

33. Perrault SD, Walkey C, Jennings T, Fischer HC, Chan WC. Mediating tumor targeting efficiency of nanoparticles through design. Nano Lett. 2009; 9: 1909-15.

34. Northfelt DW, Dezube BJ, Thommes JA, Miller BJ, Fischl MA, Friedman-Kien A, et al. Pegylated-liposomal doxorubicin versus doxorubicin, bleomycin, and vincristine in the treatment of AIDS-related Kaposi's sarcoma: results of a randomized phase III clinical trial. J Clin Oncol. 1998; 16: 2445-51.

35. Gradishar WJ, Tjulandin S, Davidson N, Shaw H, Desai N, Bhar P, et al. Phase III trial of nanoparticle albumin-bound paclitaxel compared with polyethylated castor oil-based paclitaxel in women with breast cancer. J Clin Oncol. 2005; 23: 7794-803.

36. Huo S, Ma H, Huang K, Liu J, Wei T, Jin S, et al. Superior penetration and retention behavior of $50 \mathrm{~nm}$ gold nanoparticles in tumors. Cancer Res. 2013; 73: 319-30. 
37. Tang L, Fan TM, Borst LB, Cheng J. Synthesis and biological response of size-specific, monodisperse drug-silica nanoconjugates. ACS Nano. 2012; 6: 3954-66.

38. Tang L, Gabrielson NP, Uckun FM, Fan TM, Cheng J. Size-dependent tumor penetration and in vivo efficacy of monodisperse drug-silica nanoconjugates. Mol Pharm. 2013; 10: 883-92.

39. Murphy CI, Sau TK, Gole AM, Orendorff CJ, Gao J, Gou L, et al. Anisotropic metal nanoparticles: synthesis, assembly, and optical applications. J Phys Chem B. 2005; 109: 13857-70.

40. Schindelin J, Arganda-Carreras I, Frise E, Kaynig V, Longair M, Pietzsch T, et al. Fiji: an open-source platform for biological-image analysis. Nat Methods. 2012; 9: 676-82.

41. Vigderman L, Zubarev ER. High-yield synthesis of gold nanorods with longitudinal SPR peak greater than $1200 \mathrm{~nm}$ using hydroquinone as a reducing agent. Chem Mater. 2013; 25: 1450-7.

42. Jana NR, Gearheart L, Murphy CJ. Seed-mediated growth approach for shapecontrolled synthesis of spheroidal and rod-like gold nanoparticles using a surfactant template. Adv Mater. 2001; 13: 1389-93.

43. Liebscher Jr, Mrówczyński R, Scheidt HA, Filip C, Hădade ND, Turcu R, et al. Structure of polydopamine: a never-ending story? Langmuir. 2013; 29: 10539-48.

44. Jin Z, Sugiyama Y, Zhang C, Palui G, Xin Y, Du L, et al. Rapid photoligation of gold nanocolloids with lipoic acid-based ligands. Chem Mater. 2020; 32: 7469-83.

45. Nguyen SC, Zhang Q, Manthiram K, Ye X, Lomont JP, Harris CB, et al. Study of heat transfer dynamics from gold nanorods to the environment via time-resolved infrared spectroscopy. ACS Nano. 2016; 10: 2144-51.

46. Chen YS, Frey W, Aglyamov S, Emelianov S. Environment-dependent generation of photoacoustic waves from plasmonic nanoparticles. Small. 2012; 8: 47-52.

47. Huang X, Neretina S, El-Sayed MA. Gold nanorods: from synthesis and properties to biological and biomedical applications. Adv Mater. 2009; 21: 4880-910.

48. Zhang C, Wu B-H, Du Y, Ma M-Q, Xu Z-K. Mussel-inspired polydopamine coatings for large-scale and angle-independent structural colors. J Mater Chem C. 2017; 5: 3898-902.

49. Repenko T, Rix A, Nedilko A, Rose J, Hermann A, Vinokur R, et al. Strong photoacoustic signal enhancement by coating gold nanoparticles with melanin for biomedical imaging. Adv Funct Mater. 2018; 28: 1705607.

50. Mei Z, Gao D, Hu D, Zhou H, Ma T, Huang L, et al. Activatable NIR-II photoacoustic imaging and photochemical synergistic therapy of MRSA infections using miniature Au/Ag nanorods. Biomaterials. 2020; 251: 120092.

51. Deng X, Liang S, Cai X, Huang S, Cheng Z, Shi Y, et al. Yolk-shell structured $\mathrm{Au}$ nanostar@ metal-organic framework for synergistic chemo-photothermal therapy in the second near-infrared window. Nano lett. 2019; 19: 6772-80. 